\title{
Atomic Scale Compositional and Structural Characterization of Nanostructured Materials Using Combined FIB, STEM, and LEAP
}

\author{
Brian P. Gorman*, David Diercks*, Michael Kaufman*, Rob Ulfig**, Dan Lawrence**, Keith \\ Thompson**, and David J. Larson** \\ * Department of Materials Science and Engineering, University of North Texas, Denton, TX, 76203 \\ ** Imago Scientific Instruments, Madison, WI 53719
}

Most nanoscale materials require both structural and compositional characterization in order to be able to predict fully their electrical, magnetic, or optoelectronic behaviors. Transmission electron microscopy can now give sub- $\AA$ structural information, but low-level chemical sensitivity is still lacking. Three-dimensional atom probe (3DAP) can give atomic level compositional information, but the data needs to be reconstructed to achieve the structural information [1]. Due to this complementary data acquired from TEM and 3DAP, preparation techniques that allow specimens to be examined in both the TEM and the local electrode atom probe (LEAP) have been developed. As noted in other work [2-4], FIB preparation allows for site specific specimens to be lifted out and sharpened to a diameter of less than $100 \mathrm{~nm}$ in-situ. In this study, the FIB was used to mount specimens to a TEM grid, followed by direct examination in the TEM. Using high resolution TEM structural information such as material geometry, tip radius, shank angle and d-spacing, the LEAP data reconstruction algorithms can be tuned to match the structure. Compositional information obtained using STEM-EDS and STEM-EELS is also used to compare the techniques.

In this study, metallic multilayers (PtMn / CoFe) deposited on Si posts were examined. The post structures were mounted to TEM grids in-situ using Pt GIS within a FEI Nova 200 FIB equipped with an Omniprobe Autoprobe 200 nanomanipulator (Fig. 1). Specimens were subsequently examined by TEM in an FEI Tecnai G2 F20. Fig. 2 is a Z-contrast (STEM-HAADF) image of the FIB processed multilayer structure. Good contrast is observed between the different layers in the structure, which allows for careful geometric measurements. EDS spectra were acquired in STEM mode using a $1 \mathrm{~nm}$ probe at $200 \mathrm{keV}$ accelerating voltage and $4500 \mathrm{~V}$ extractor voltage using a $100 \mu \mathrm{s}$ time constant. EELS spectra were acquired in parallel with the HAADF and EDS using a Gatan Tridiem PEELS/GIF. Fig. 3 shows the STEM-EDS compositional profile acquired at $1 \mathrm{~nm}$ step resolution and a $5 \mathrm{sec}$ dwell time. Although variations in the composition are observable, the data has significant noise, most likely due to beam broadening and X-ray fluorescence. EELS data is less noisy and has higher spatial resolution than EDS, but still lacks low level compositional information. Figure 4 is a 3-D LEAP reconstruction of a multilayer sample. Following data reconstruction, the structural information obtained from the LEAP is similar to that obtained in the TEM. A 1-D composition profile of the atom-probe data is obtained by creating a cylinder in the analysis direction, slicing the cylinder into $0.1 \mathrm{~nm}$ slices, and counting the atoms in each slice. This data demonstrates the much improved statistics of LEAP over STEM-EDS. Further comparisons of the compositional and structural data will be detailed, as will refinements to the LEAP data reconstruction using correlating TEM data.

[1] T. F. Kelly and D. J. Larson, Mat. Char. 44 (2000) 59.

[2] D. J. Larson et al., Nanotech. 10 (1999) 45.

[3] D. J. Larson et al., Micro. Microanal. 7 (2001) 24.

[4] M. K. Miller, K. F. Russell and G. B. Thompson, Ultramicroscopy 102 (2005) 287. 


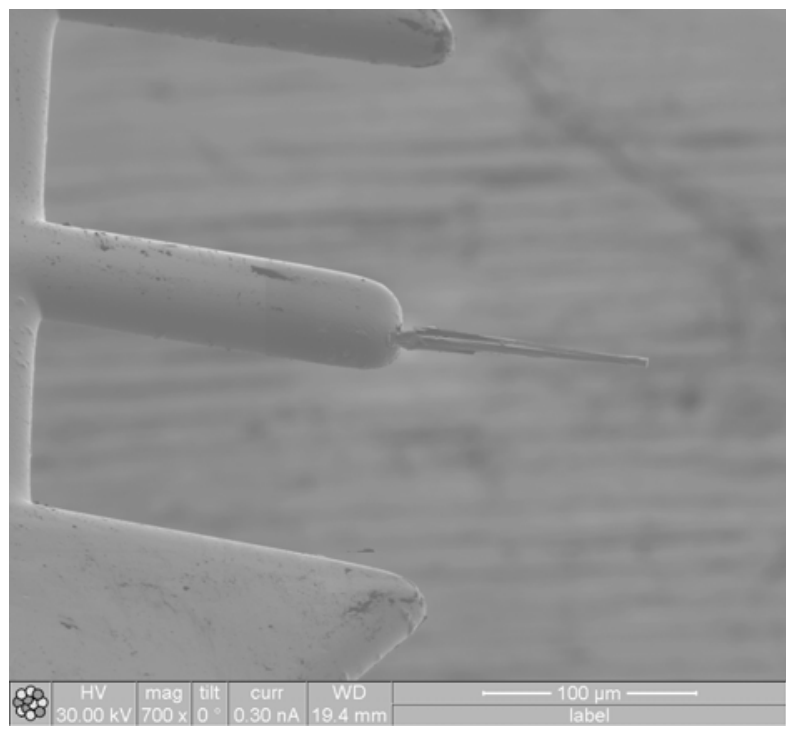

Fig. 1. SEM image of multilayer thin film structure deposited on $\mathrm{Si}$ microposts and subsequently mounted to a TEM grid.

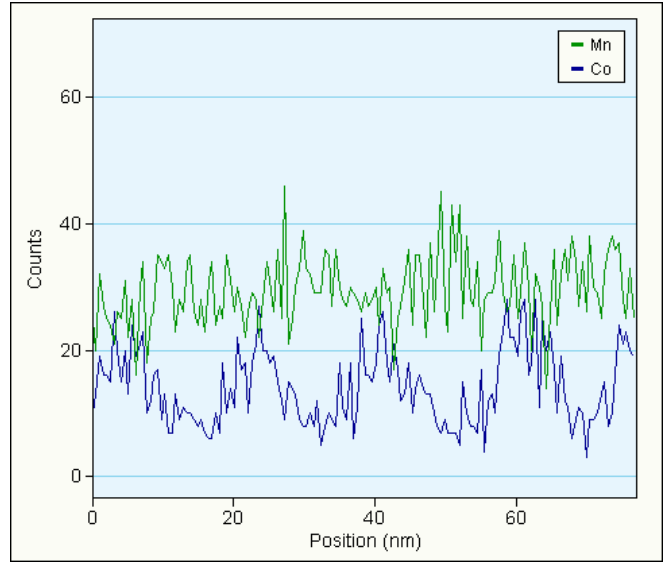

Fig 3. STEM-EDS compositional profile obtained from the atom probe specimen.

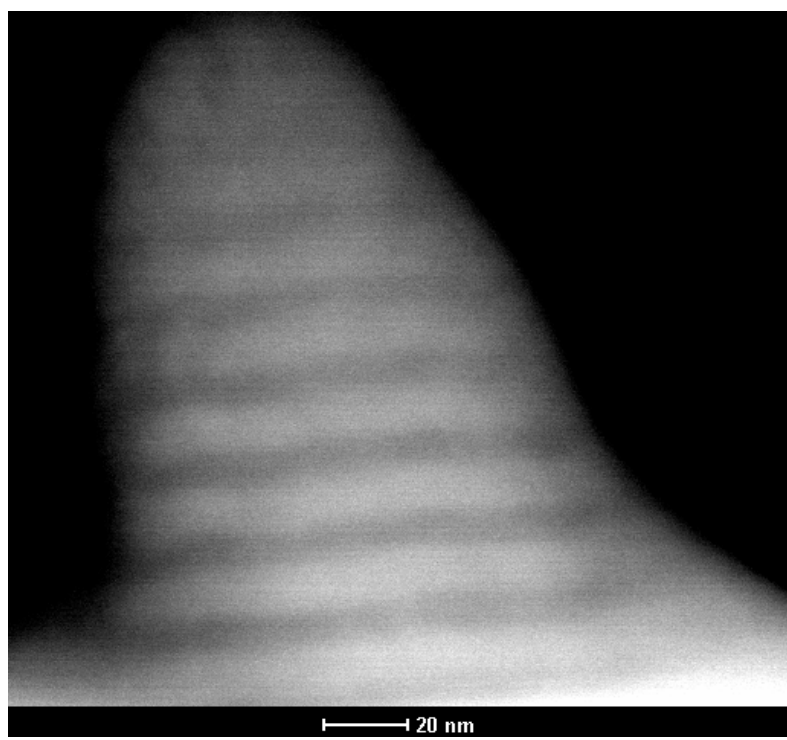

Fig. 2. STEM-HAADF image of a multilayer thin film prepared as a LEAP specimen by FIB.

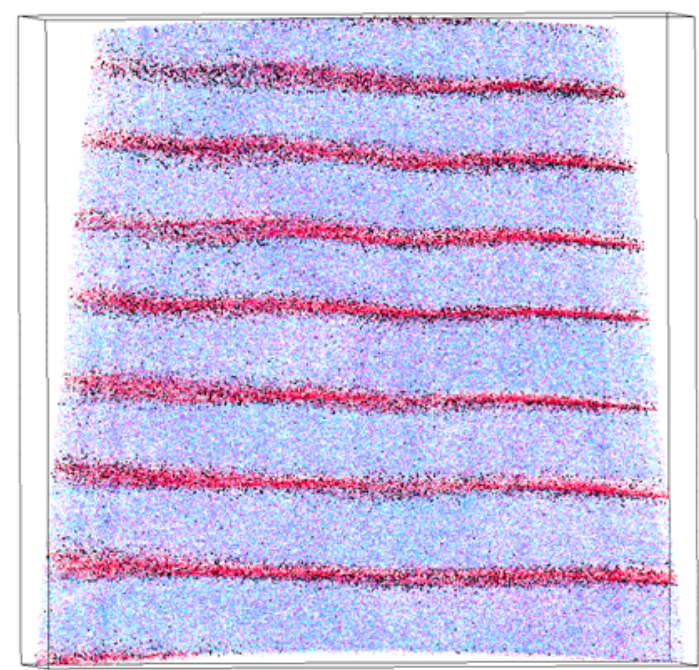

Fig 4. LEAP 3-D reconstruction of the multilayer thin film specimen. $\mathrm{FOV}=150 \mathrm{x}$ $70 \mathrm{~nm}$

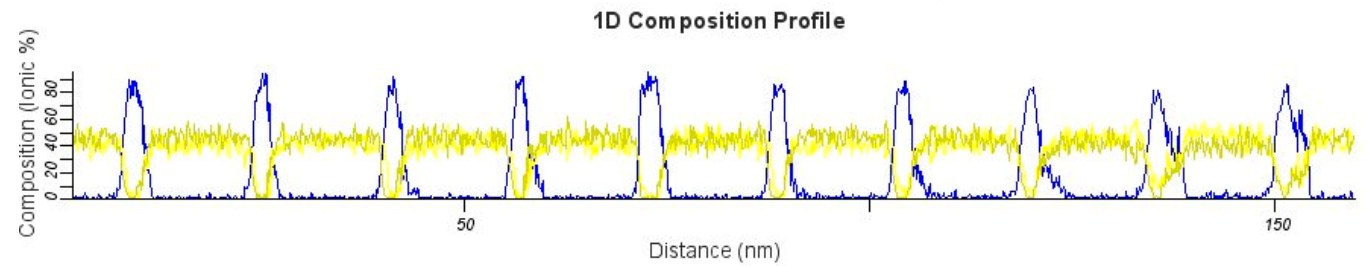

Fig 5. 1-D compositional profile obtained from a $5 \mathrm{~nm}$ diameter selected perpendicular to the layers. Yellow $=\mathrm{Pt}$, Blue $=\mathrm{Co}$ 